\section{Clinical characteristics and prognostic factors of scleral rupture due to blunt ocular trauma}

OE Yucel1', S Demir², L Niyaz¹ , O Sayin³ ${ }^{3}$ A Gul ${ }^{1}$ and $\mathrm{N}$ Ariturk ${ }^{1}$
${ }^{1}$ Department of Ophthalmology, Medical Faculty, Ondokuz Mayis University, Samsun, Turkey

${ }^{2}$ Department of Ophthalmology, Medical Faculty, Gaziosmanpasa University, Tokat, Turkey

${ }^{3}$ Clinic of ophthalmology, Develi Hatice Muammer Kocaturk State Hospital, Kayseri, Turkey

Correspondence: OE Yucel, Ondokuz Mayis University, Medical Faculty, Department of Ophthalmology, Atakum 55139, Samsun, Turkey Tel: +903623121919/ 905057191071; Fax: +903624576041 . E-mail: drozlem38@hotmail. com

Received: 27 January 2016 Accepted in revised form: 11 July 2016 Published online: 2 September 2016

\begin{abstract}
Purpose To describe and identify ocular and wound characteristics, and prognostic factors associated with final visual acuity (VA) in patients with scleral rupture due to blunt ocular trauma.

Methods The medical records of 61 patients with globe rupture due to blunt ocular trauma who underwent primary repair were reviewed retrospectively. The data recorded included demographic characteristics, initial and final VA, ocular signs, wound characteristics, and surgeries. Initial VA, ocular signs, wound characteristics, and surgeries were analyzed to determine the association with the final VA.

Results Forty three women and 18 men with a mean age of $43.6 \pm 23.5$ years were included in the study. The locations of scleral wounds were mostly in the superonasal quadrant (41.0\%) and zone 2 (75.4\%). In eyes with hyphema $(P=0.009)$, vitreous hemorrhage $(P=0.001)$, and retinal detachment $(P=0.004)$, final VA was statistically worse than eyes without these signs. A moderate positive correlation was found between the initial and final VA $(P<0.001)$. Final VA was statistically worse in eyes with horizontal midline wounds than in eyes with vertical midline wounds $(P=0.002)$. A moderate negative correlation was found between scleral wound length and final VA $(P<0.001)$. Patients who underwent cataract surgery had statistically better final VA $(P=\mathbf{0 . 0 0 2})$.

Conclusions Scleral rupture was detected mostly in females, superonasal quadrant and zone 2. Poor final VA was significantly associated with poor initial VA, longer wound length, horizontal midline wound, presence of hyphema, vitreous hemorrhage and retinal detachment at presentation, and cataract surgery not performed during followup period. Scleral ruptures have different demographic, ocular and wound characteristics than other open globe injuries.
\end{abstract}

Eye (2016) 30, 1606-1613; doi:10.1038/eye.2016.194; published online 2 September 2016

\section{Introduction}

Open globe injuries (OGIs) are a major cause of visual loss and blindness. The mean estimated incidence of OGIs is $~ 3.5$ cases in 100000 annually, leading to 200000 OGIs per year worldwide. ${ }^{1}$ A globe rupture is a type of OGI caused by blunt ocular trauma and has poor visual outcome. ${ }^{2}$

Several studies have suggested that the factors that significantly predict visual outcome after OGIs are presenting visual acuity (VA), ${ }^{3-6}$ presence of a relative afferent pupillary defect (RAPD), ${ }^{3,7-9}$ mechanism of injury, ${ }^{4,6,7,10,11}$ wound location, $, 8,10,12-14$ wound size and extension, 10,11,15,16 adnexal trauma, 7,9,12,17 lens damage, ${ }^{4,11}$ hyphema, ${ }^{3,11}$ vitreous hemorrhage, $3,4,8,10,12$ and retinal detachment. ${ }^{4,5,7,12,17}$

The major signs of scleral rupture are VA with light perception or less, conjunctival edema and subconjunctival hemorrhage, hyphema, ocular hypotony, impairment or dislocation of the lens, vitreous hemorrhage, and retinal detachment. ${ }^{15,16,18}$ To our knowledge, there are not enough studies on the clinical characteristics and their effects on final VA in eyes with scleral rupture. Russell et al determined that an initial VA of 5/200 or beter, absence of scleral rupture, and a rupture length of less than $11 \mathrm{~mm}$ were prognostic factors of ambulatory vision for eyes with ruptured and intact globes after blunt trauma. ${ }^{15}$ The absence of a hyphema and the presence of a rupture of $9 \mathrm{~mm}$ or less were reported as good prognostic signs. ${ }^{16}$

In the present study, we aimed to describe and identify ocular and wound characteristics and prognostic factors associated with final VA in patients with scleral rupture due to blunt ocular trauma. 


\section{Materials and methods}

The medical records of patients with OGIs who underwent primary repair between January 2008 and December 2013 were reviewed retrospectively. Sixty one eyes of 61 patients with scleral rupture due to blunt ocular trauma were included in the study. This study was approved by the local research ethics committee.

The data recorded included demographic characteristics, initial and final VA, ocular signs (hyphema, endophthalmitis, uveal tissue prolapse or damage, dislocation of the lens, phacocele, traumatic cataract, vitreous hemorrhage, and retinal detachment), wound characteristics (location, direction, and length), and surgeries.

The type and zone of injury were defined according to the classification system of the Ocular Trauma Classification Group. ${ }^{2}$ Globe rupture was defined as a full thickness wound of the eye wall involving the sclera with or without the cornea due to blunt ocular trauma. The location of injury was defined as zone 1 (wound involvement limited to the cornea, including corneoscleral limbus), zone 2 (wound involving $5 \mathrm{~mm}$ of sclera from the corneoscleral limbus), or zone 3 (wound involving the sclera posterior to the $5 \mathrm{~mm}$ from the corneoscleral limbus). Zone 1 injuries were excluded from the study because of the absence of scleral wound. The location of injury was additionally defined as superonasal quadrant, inferonasal quadrant, superotemporal quadrant, inferotemporal quadrant, horizontal midline, and vertical midline. According to the direction to the limbus, the wound was defined as parallel, perpendicular or angled, or both. Location and direction of the scleral rupture are illustrated in Figure 1. The lengths of scleral and corneal wounds were recorded from charts of the globe repair.

In this study, presenting and final VA were grouped (1 through 5) as no light perception (NLP), light perception (LP)/hand motion (HM), 1/200 to $19 / 200$, $20 / 200$ to $20 / 50$, and $\geq 20 / 40$, similar to the Ocular Trauma Score study group. ${ }^{19}$

In addition to the zone 1 injuries, penetrating and perforating injuries, cases with missing records and those with a follow-up period of less than 6 months were excluded from the study.

\section{Statistical methods}

The following variables were analyzed to determine the association with final VA: initial VA, wound length, wound location, wound direction, presence of corneal wound, dislocation of lens or phacocele, uveal tissue prolapse or damage, hyphema, vitreous hemorrhage and retinal detachment at presentation, traumatic cataract, performance of vitrectomy and cataract surgery during follow-up period.

The statistical analysis was performed using V21 IBM SPSS (SPSS Inc., Chicago, IL, USA). The Shapiro-Wilk test was used as a normality test for the quantitative data. The Kruskal-Wallis and Mann-Whitney U tests were used to compare non-normally distributed data. The MannWhitney $U$-test with Bonferroni correction was used to determine the source of the differences seen in the Kruskal-Wallis test. The $\chi^{2}$-test was used to compare categorical data. The relationships between variables were analyzed with Spearman's correlation. The results were presented as median (min-max), frequency (percent), and mean \pm SD. A $P$-value of $<0.05$ was considered statistically significant. A $P$-value of $<0.003$ was considered statistically significant for the MannWhitney $U$-test with Bonferroni correction.

\section{Results}

Sixty one eyes of 61 patients with scleral rupture due to blunt ocular trauma were included in the study. Fortythree $(70.5 \%)$ were women, and $18(29.5 \%)$ were men. The mean age was $43.6 \pm 23.5(5-80)$ years. The mean follow up period was $11.5 \pm 9.4$ (6-36) months.

Slit-lamp biomicroscopic examination revealed subconjunctival hemorrhage (61/61, 100\%), hyphema $(58 / 61,95.1 \%)$, dislocation of the lens or phacocele $(13 / 61,20.6 \%)$, and uveal tissue prolapse or damage $(32 / 61,52.4 \%)$ at presentation. The hyphema was partial in $24(39.3 \%)$ and total in $34(55.7 \%)$ of 61 eyes. Relative afferent pupillary defect could not be assessed because of the dense or full hyphema in these eyes. X-ray computed tomography (CT) was performed on all patients before the operation. B-scan ultrasonography was done in all cases after the primary operation. Vitreous hemorrhage was detected in 43 (70.5\%) of 61 eyes, and retinal detachment was detected in $25(40.9 \%)$ of 61 eyes by ultrasonography. None of the eyes had an intraocular foreign body. There were no cases of acute endophthalmitis. The clinical characteristics of the patients can be seen in Table 1.

Initial VA ranged from NLP $(n=20,32.8 \%)$ to $20 / 60$ $(n=1,1.6 \%)$. Final VA ranged from NLP $(n=26,42.6 \%)$ to $20 / 20(n=3,4.9 \%)$. The distribution of initial and final $\mathrm{VA}$ can be seen in Table 1.

There were 46 (75.4\%) zone 2 and 25 (24.6) zone 3 injuries. Scleral rupture was present in the right eye in $28(45.9 \%)$ of 61 cases and in the left eye in $33(54.1 \%)$ of 61 cases. The locations of scleral wounds were mostly in the superonasal quadrant $(25 / 61,41.0 \%)$; other locations can be seen in Table 1 . The wound was parallel in 27 $(44.3 \%)$ cases, perpendicular or angled in 28 (45.9\%), and both in $6(9.8 \%)$ of 61 eyes. There was a corneal 


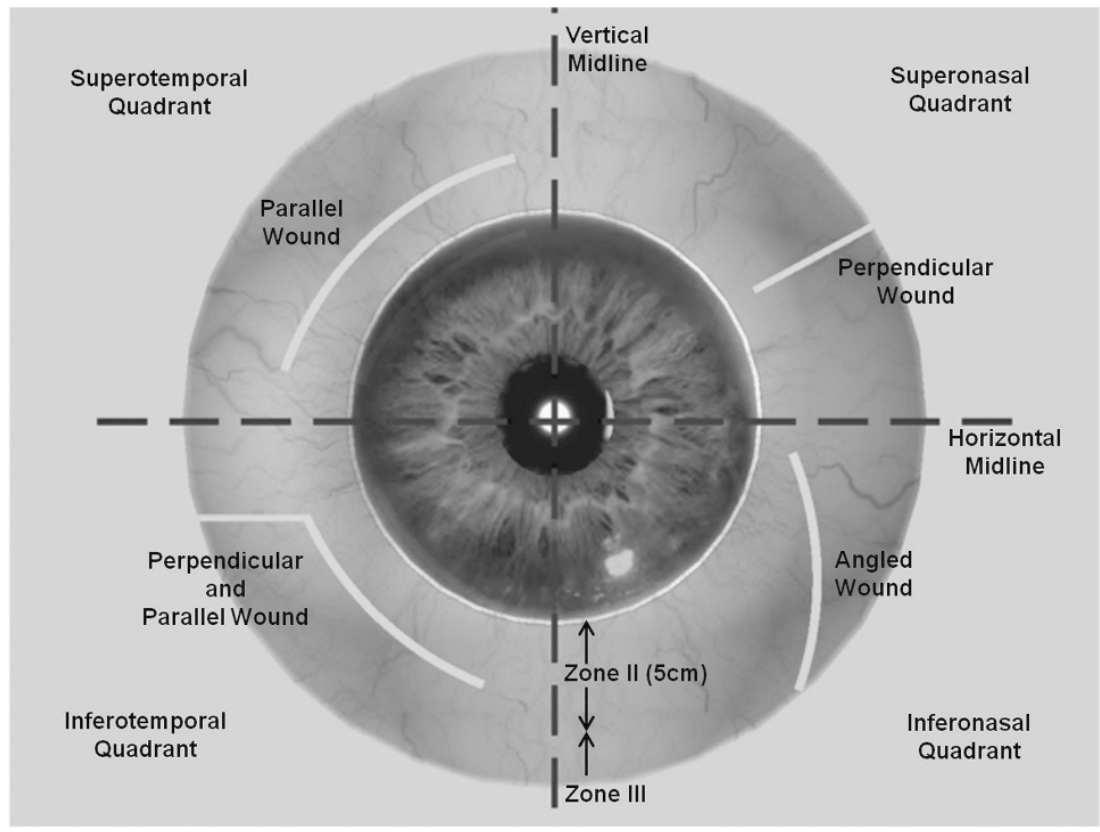

Figure 1 Location and direction of the scleral rupture are illustrated on the right eye.

involvement of wound in $24(39.3 \%)$ of 61 eyes. Sixteen $(34.8 \%)$ eyes with zone 2 and 8 (\%53.3) eyes with zone 3 injuries had corneal involvement. The lengths of scleral and total (sclera+cornea) wounds were $9.04 \pm 5.9$ and $12.42 \pm 5.7 \mathrm{~mm}$, respectively.

All the eyes underwent primary globe repair. The mean time duration between injury and primary globe repair was $6.85 \pm 3.3(3-16) \mathrm{h}$. None of the eyes were primarily eviscerated or enucleated. Eleven (18.0\%) of 61 eyes underwent secondary evisceration due to painful blind eye. Sixteen $(26.2 \%)$ of 61 eyes underwent cataract surgery, and $8(13.1 \%)$ of 61 eyes underwent vitreoretinal surgery after primary globe repair.

The rate of phthisical eye was $24.6 \%$ (15/61) during the follow up period. The hyphema was partial in $13.3 \%$ $(2 / 15)$ and total in $86.7 \%(13 / 15)$ of phthisical eyes. Vitreous hemorrhage and retinal detachment were detected in $100 \%(15 / 15)$ and $86.7 \%(13 / 15)$ of phthisical eyes, respectively. Initial VA was NLP in $66.7 \%(10 / 15)$ and $\mathrm{LP} / \mathrm{HM}$ in $33.3 \%(5 / 15)$ of phthisical eyes.

\section{Visual outcome}

The initial clinical signs were analyzed to determine which factors were associated with poor final VA. In eyes with hyphema $(P=0.009)$, vitreous hemorrhage $(P=0.001)$, and retinal detachment $(P=0.004)$, final VA was statistically worse than eyes without these signs. An association between uveal tissue prolapse or damage, dislocation of the lens or phacocele and final VA was not detected. A moderate positive correlation was found between the initial and final VA (Correlation

Coefficient $=0.588, P<0.001$ ), as seen in Table 1 .

Final VA was statistically worse in eyes with horizontal midline wounds than in eyes with vertical midline wounds $(P=0.002)$. There were no significant associations between other wound locations and final VA. An association between zone of injury, wound direction, corneal wound presence and final VA was not detected, as seen in Table 1. A moderate negative correlation was found between scleral, total wound lengths and final VA (Correlation Coefficient $=-0.496$ and -0.417 , and $P<0.001$ and $P=0.001$, respectively).

There was no significant association between between final VA and time duration between injury and primary globe repair $(P=0.383)$. In eyes that underwent cataract surgery, final VA was statistically better $(P=0.002)$, but an association between vitreoretinal surgery and final VA was not detected, as seen in Table 1.

Total hyphema $(P=0.01)$, vitreous hemorrhage $(P=0.01)$, retinal detachment $(P=0.001)$, and initial VA of NLP $(P=0.007)$ were factors predictive of phthisical eyes. An association between phthisical eyes and other ocular and wound characteristics was not detected.

\section{Discussion}

Numerous studies have been published to correlate final visual outcome with several factors related to OGIs.

To our knowledge, there are not enough studies on the clinical characteristics and their effects on final VA in eyes with scleral rupture. This study identified associations 
Table 1 Clinical characteristics and visual prognosis of patients with scleral rupture $(n=61)$

\begin{tabular}{|c|c|c|c|c|c|c|c|}
\hline \multirow[t]{2}{*}{ Variable } & \multicolumn{5}{|c|}{ Final visual acuity } & \multirow[t]{2}{*}{ Total } & \multirow[t]{2}{*}{$\mathrm{P}$} \\
\hline & $N L P$ & $L P / H M$ & $1 / 200-19 / 200$ & $20 / 200-20 / 50$ & $\geq 20 / 40$ & & \\
\hline \multicolumn{8}{|l|}{ Gender } \\
\hline Female & 18 & 11 & 3 & 5 & 6 & 43 & 0.885 \\
\hline Male & 7 & 5 & 2 & 1 & 3 & 18 & \\
\hline \multicolumn{8}{|l|}{ Eye } \\
\hline Right & 16 & 5 & 2 & 3 & 2 & 28 & 0.112 \\
\hline Left & 9 & 11 & 3 & 3 & 7 & 33 & \\
\hline \multicolumn{8}{|l|}{ Zone of injury } \\
\hline Zone 1 & 15 & 14 & 3 & 6 & 8 & 46 & 0.213 \\
\hline Zone 2 & 10 & 2 & 2 & 0 & 1 & 15 & \\
\hline \multicolumn{8}{|l|}{ Location of injury } \\
\hline Superonasal & 3 & 4 & 0 & 1 & 1 & 9 & $0.005^{\mathrm{a}}$ \\
\hline Superotemporal & 1 & 4 & 1 & 0 & 1 & 7 & \\
\hline Inferonasal & 11 & 5 & 2 & 4 & 3 & 25 & \\
\hline Inferotemporal & 3 & 1 & 1 & 0 & 0 & 5 & \\
\hline Horizontal midline & 1 & 0 & 0 & 1 & 4 & 6 & \\
\hline Vertical midline & 6 & 2 & 1 & 0 & 0 & 9 & \\
\hline \multicolumn{8}{|l|}{ Direction of injury } \\
\hline Parallel & 10 & 9 & 1 & 4 & 3 & 27 & 0.782 \\
\hline Perpendicular or angled & 10 & 7 & 4 & 2 & 5 & 28 & \\
\hline Both of them & 5 & 0 & 0 & 0 & 1 & 6 & \\
\hline \multicolumn{8}{|l|}{ Corneal involvement } \\
\hline Yes & 9 & 6 & 2 & 2 & 5 & 24 & 0.541 \\
\hline No & 16 & 10 & 3 & 4 & 4 & 37 & \\
\hline \multicolumn{8}{|l|}{ Hyphema } \\
\hline Total & 20 & 9 & 1 & 1 & 3 & 34 & $0.009^{\mathrm{a}}$ \\
\hline Partial & 4 & 7 & 3 & 5 & 5 & 24 & \\
\hline No & 1 & 0 & 1 & 0 & 1 & 3 & \\
\hline \multicolumn{8}{|c|}{ Dislocation of the lens or phacocele } \\
\hline Yes & 4 & 4 & 1 & 4 & 0 & 13 & 0.830 \\
\hline No & 21 & 12 & 4 & 2 & 9 & 48 & \\
\hline \multicolumn{8}{|l|}{ Uveal tissue prolapse or damage } \\
\hline Yes & 16 & 8 & 3 & 2 & 3 & 32 & 0.164 \\
\hline No & 9 & 8 & 2 & 4 & 6 & 29 & \\
\hline \multicolumn{8}{|l|}{ Vitreous hemorrhage } \\
\hline Yes & 25 & 10 & 2 & 2 & 4 & 43 & $0.001^{\mathrm{a}}$ \\
\hline No & 0 & 6 & 3 & 4 & 5 & 18 & \\
\hline \multicolumn{8}{|l|}{ Retinal detachment } \\
\hline Yes & 19 & 2 & 3 & 0 & 1 & 25 & $0.004^{\mathrm{a}}$ \\
\hline No & 6 & 14 & 2 & 6 & 8 & 36 & \\
\hline \multicolumn{8}{|l|}{ Cataract surgery } \\
\hline Yes & 0 & 5 & 2 & 5 & 4 & 16 & $0.002^{\mathrm{a}}$ \\
\hline No & 25 & 11 & 3 & 1 & 5 & 45 & \\
\hline \multicolumn{8}{|l|}{ Vitreoretinal surgery } \\
\hline Yes & 0 & 3 & 2 & 1 & 2 & 8 & 0.349 \\
\hline No & 25 & 13 & 3 & 5 & 7 & 53 & \\
\hline
\end{tabular}


Table 1 (Continued)

\begin{tabular}{|c|c|c|c|c|c|c|c|}
\hline \multirow[t]{2}{*}{ Variable } & \multicolumn{5}{|c|}{ Final visual acuity } & \multirow[t]{2}{*}{ Total } & \multirow[t]{2}{*}{$\mathrm{P}$} \\
\hline & $N L P$ & $L P / H M$ & $1 / 200-19 / 200$ & $20 / 200-20 / 50$ & $\geq 20 / 40$ & & \\
\hline \multicolumn{8}{|l|}{ Initial visual acuity } \\
\hline NLP & 18 & 0 & 0 & 0 & 1 & 19 & \multirow{5}{*}{$<0.001^{\mathrm{a}}$} \\
\hline $\mathrm{LP} / \mathrm{HM}$ & 7 & 16 & 5 & 5 & 5 & 38 & \\
\hline $1 / 200-19 / 200$ & 0 & 0 & 0 & 1 & 2 & 3 & \\
\hline $20 / 200-20 / 50$ & 0 & 0 & 0 & 0 & 1 & 1 & \\
\hline$\geq 20 / 40$ & 0 & 0 & 0 & 0 & 0 & 0 & \\
\hline
\end{tabular}



between wound and ocular characteristics, surgeries and final VA in patients with scleral rupture due to blunt ocular trauma.

Open globe injuries occur most frequently in males. ${ }^{3,5-9,12}$ In contrast to the literature, a significant female predominance was found in our scleral rupture series. Emami-Naeini et al ${ }^{14}$ reported a female predominance in fall-related OGIs. In addition, in their series, the most common type of injury was rupture (90\%). Because of this; we proposed that globe rupture may affect female patients more. Our hospital is a regional hospital that serves both rural and urban areas. In this region, women also take an active role in working life. Especially, in rural areas, women are working in the fields and farming. Therefore, women are more exposed to work accidents. Open globe injuries can be observed in all age groups, especially between 30 and 50 years old. $3,5,7,9,11,12$ In our study, the mean age was $43.6 \pm 23.5$ (5-80) years, and this is similar to previous reports.

The rates of patients with VA of NLP were 32.8 and $42.6 \%$ at initial and final visits. Bi et al reported the rates of patients with VA of NLP and $<0.1$ were $30.7 \%$ and $100 \%$, respectively, at initial presentation in their ruptured globe injury series. The ratio of better VA (better than 0.1 ) increased from 0 preoperatively to $16.0 \%$ postoperatively, and VA improved in approximately half of the patients. ${ }^{20}$ We found a moderate-positive correlation between the initial and final VA. Poor initial VA was a strong predictor of poor visual outcome $(P<0.001)$. Visual acuity changed from NLP to $20 / 32$ in only one patient $(1 / 19,5.2 \%)$. In 7 patients $(7 / 42,16.7 \%)$, VA changed from LP or better to NLP. Visual recovery rate from NLP to LP or better for OGIs varies in the literature; Agrawal et al, Salehi-Had et al, and Soni et al reported it in 33.3\%, $26.1 \%$, and $23 \%$ of their patients, respectively. ${ }^{8,21,13}$

Feng et al reported a $54.5 \%$ visual recovery rate in patients who underwent vitreoretinal surgery. ${ }^{10}$

In our series, hyphema was seen in $95.1 \%$ of patients. Russel et $a^{15}$ reported that the rate of hyphema was $78.9 \%$ in eyes with occult ruptures. This rate was reported to be
20.1 to $100 \%$ in severe OGIs in the literature. $5,7,8,10,13,14$ Hyphema was a prognostic factor for poor final VA $(P=0.009)$ in our study. Rofail et $a l^{3}$ reported hyphema was a predictor of poorer visual outcome. Although, in some studies, multiple regression analysis demonstrated that there was no correlation between hyphema and final VA. 5,7

Dislocation of the lens or phacocele was seen in $20.6 \%$ of patients. Sixteen $(26.2 \%)$ patients underwent cataract surgery. In the literature, there are studies that have reported similar rates of lens injury and traumatic cataract $^{8,17}$ and studies that have reported higher rates of lens injury and traumatic cataract. ${ }^{4,6,12}$ Patients who underwent cataract surgery had statistically better final VA $(P=0.002)$ in our study. Al-Mezaine et $a l^{4}$ said that poor final visual outcome was associated with cataract surgery if it was not performed during the follow-up period. In contrast, some studies detected no association between lens injury or traumatic cataract and visual outcome. .,8,12 $^{6}$

Uveal tissue prolapse or damage was seen in $52.4 \%$ of patients. In contrast to the results of some studies, uveal tissue prolapse or damage was not a predictor of visual outcome in our series. In the series by Rofail et al, ${ }^{3}$ iris prolapse occurred in $47.6 \%$ of patients, and it was a predictor of poor visual outcome. Likewise, in the series by Yalcin Tök et al, ${ }^{5}$ uveal tissue prolapse occurred in $21.7 \%$ of patients, and it adversely affected final VA.

Bi et al reported choroidal injuries in $62.7 \%$ of patients with ruptured globe injuries. They said that choroidal injury could result in severe hemorrhage, scarring, and proliferation. ${ }^{20}$ We did not use ultrasound biomicroscope to determine ciliary or choroidal injuries. We detected high rates of vitreous hemorrhage and VA of NLP. This could be a result of ciliary or choroidal injuries. The rate of vitreous hemorrhage was $70.5 \%$ in our series. Similar to the results of many studies, vitreous hemorrhage was a prognostic factor for poor final VA $(P=0.001)$. In the study by Rofail et al, ${ }^{3}$ vitreous hemorrhage was seen in $40.6 \%$ of patients and was reported as a predictor of poor 
visual outcome. In the study by Al-Mezaine et al ${ }^{4}$ vitreous hemorrhage was seen in a small number of patients $(6.5 \%)$, but it had a significant association with final visual outcome. The rate of vitreous hemorrhage was $77.8 \%$ in eyes with NLP in the study by Agrawal et al. They found an association between vitreous hemorrhage and poor postoperative outcome. ${ }^{8}$ However, some studies found no association between vitreous hemorrhage and visual outcome. ${ }^{6,13,14}$ Feng et al ${ }^{10}$ considered that severe intraocular hemorrhage was not only a predictor of an unfavorable outcome, but also a reflection of the seriousness of the ocular damage, which includes severe intraocular tissue injury, such as to the choroid and retina.

In our study, retinal detachment was detected in $40.9 \%$ of patients. Retinal detachment was a prognostic factor for poor final VA $(P=0.004)$. In the study by Feng et al ${ }^{10}$ retinal detachment was seen in $51.5 \%$ of patients whose vision was NLP, and it was a prognostic factor for outcome. There are studies reporting lower retinal detachment rates and significant association between retinal detachment and final VA. ${ }^{4,5,7}$ Conversely, some studies reported that retinal detachment was not a predictor of outcome. 6,8,13,14

An association between vitreoretinal surgery and final VA was not detected in our study. Al-Mezaine et al reported that performance of pars plana vitrectomy was a significant negative predictor for good final VA but was a significant positive predictor for an intermediate visual outcome. ${ }^{4}$ Emami-Naeini et al ${ }^{14}$ suggested PPV had a role in preventing and/or diminishing the risk of developing NLP in posterior segment complications associated with fall-related OGIs. Feng et al ${ }^{10}$ said that traumatized eyes with NLP can be anatomically restored with LP or better vision if vitreoretinal surgery is attempted. Russell et al ${ }^{15}$ said that the vitrectomized eyes had a better result, suggesting that early pars plana vitrectomy is of benefit in selected rupture cases.

Eleven $(18.0 \%)$ eyes underwent secondary evisceration in our series. The rate of evisceration-enucleation was compatible with some studies $3,9,12,17$ but was lower than the $53 \%$ in the study of Soni et al However, in their study, all eyes had NLP of VA. ${ }^{13}$

There was no case of acute endophthalmitis in our study. The incidence of post traumatic endophthalmitis varied from 1.2 to $30 \%$ in previous OGI series. $5,14,17,22$ The risk factors for endophthalmitis are presence of intraocular foreign body, trauma in a rural environment, delayed primary surgery, delayed systemic antibiotic treatment for $>24 \mathrm{~h}$, lens disruption, low age, low visual acuity at presentation, and long duration of hospital stay in OGIs. ${ }^{22-24}$ Thompson et $a l^{23}$ reported that

endophthalmitis did not occur in eyes with blunt ocular injury, which is similar to our results. Emami-Naeini et al reported an extremely low rate of endophthalmitis (1.2\%) in fall-related OGIs in which rupture is the most common type of injury $(90 \%){ }^{14}$

In our study, all injuries were zone 2 and 3 injuries. In contrast to most previous reports, there was not a significant association between zone of injury and final VA in our study. These studies reported that final visual outcomes were significantly correlated with the zone of injury. Greater posterior wound extension leads to poorer final outcomes, and eyes with zone 3 injuries were more statistically associated with poor VA. 2,4,6,8,10 Yalcin Tok et $a l^{5}$ found that the risk of having a final VA worse than 20/200 was three times higher in zone2 injuries and 5.5 times higher in zone 3 injuries when compared with zone 1 injuries. Posterior segment injury can cause irreversible damage to the retina and optic nerve and thus has a poor visual prognosis. In the study by Al-Mezaine et $a l^{4}$ OGIs involving zones 2 and 3 resulted in significantly higher rates of retinal detachments and phthisis than those involving zone 1 . In the study by Agrawal et al cases with zone 3 injuries not extending beyond 'annulus of Zinn' were associated with good outcomes. The possible explanation for adverse outcomes in injuries extending beyond 'annulus of Zinn' is significant vitreo-retinal trauma associated with posteriorly extending scleral laceration. They said that based on the justification given by Ocular Trauma Classification Group, they have arbitrarily taken it as $5 \mathrm{~mm}$ as injuries in that zone may not extend into pars plana. ${ }^{11}$ We did not evaluate the relationship between visual outcome and extent of wound according to the rectus muscle insertion.

The locations of scleral wounds were mostly in the superonasal quadrant $(41.0 \%)$ in our study. Russel et al found that the rupture occurred superiorly in 18 of 22 eyes with severe blunt ocular trauma. They reported that rupture was detected mostly in the superotemporal quadrant. ${ }^{15}$ In Cherry's report, 33 of the 41 anterior ruptures were located in the superior half of the eye. The nasal-superior quadrant between the limbus and spiral of Tillaux, the temporo-superior quadrant between the spiral of Tillaux and $5 \mathrm{~mm}$ posterior to the equator, and the temporosuperior quadrant between the limbus and spiral of Tillaux were the three most common areas involved, in decreasing order of frequency. ${ }^{16}$ Most of the scleral ruptures involved the superior hemisphere of the globe. We detected worse final VA in eyes with horizontal midline wound than in eyes with vertical midline wound $(P=0.002)$ and no significant associations with other wound locations and final VA. This might be explained by the rupture of long ciliary arteries and nerves, which may increase hemorrhage and delay or deteriorate wound healing.

According to the direction to the limbus, the wound was parallel in $44.3 \%$, perpendicular or angled in $45.9 \%$, 
and both in $9.8 \%$ of eyes. There was not a significant association between wound direction and final VA in our study. Russel et $a l^{15}$ found circumferentially oriented rupture in $36 \%$, radially oriented rupture in $28 \%$, and both circumferentially and radially oriented rupture in $36 \%$ of 25 patients. Agrawal et al reported that length of laceration or radial extent of scleral wound significantly affected the final vision outcome in zone 3 penetrating or blunt injuries. They thought that the radial scleral lacerations extending beyond rectus insertions were often associated with poorer outcome because of co-morbid factors such as retinal detachment, vitreous traction, and significant vitreous loss. ${ }^{11}$ To our knowledge, there are no studies in the literature about wound location and direction and their effects on final VA in the scleral rupture due to blunt ocular trauma.

There was a corneal involvement of wound in 39.3\% of eyes in our study. There was no significant association between presence of corneal wound and final VA. Lee et al reported that cornea wound across the pupil was a poor prognostic factor. They thought that the central location and irregularity of the corneal wound, instead of the length, would result in scarring and significant astigmatism and correlate with an unfavorable outcome. But in their study, penetrating injuries were the major type of injury. ${ }^{25}$

A moderate negative correlation was found between wound length and final VA in this study. Russel et al ${ }^{15}$ reported that final VA correlated inversely to the length of the rupture. Feng et al and Cherry detected that wound length of $9 \mathrm{~mm}$ or less was associated with good visual outcome and that wound length of $\geq 10 \mathrm{~mm}$ was associated with poor visual outcome. ${ }^{10,16}$ Al-Mezaine et al detected that wound length of $10 \mathrm{~mm}$ or less was associated with good visual outcome and that wound length of $>10 \mathrm{~mm}$ was associated with poor visual outcome. ${ }^{4}$ Pieramici et al said that larger wounds tend to involve more posterior structures and the prognostic implication of wound length is probably the posterior extent of the injury. As such, they felt that the identification of wound location was the more important factor and that additionally reporting wound length would be redundant. ${ }^{2}$ But, some ruptures are parallel to the corneoscleral limbus and not extent to the posterior of the globe.

In this study, rupture was detected mostly in females, superonasal quadrant and zone 2. Poor final VA was significantly associated with poor initial VA, longer wound length, horizontal midline wound, presence of hyphema, vitreous hemorrhage and retinal detachment at presentation, and cataract surgery not performed during follow-up period. A moderate positive correlation was found between initial and final VA, and a moderate negative correlation was found between wound lengths and final VA. Scleral ruptures have different demographic, ocular and wound characteristics than other OGIs. The careful assessment of prognostic factors of the scleral rupture due to blunt ocular trauma allows realistic expectations of final visual outcome by doctors and patients.

\section{Summary}

\section{What was known before}

- Numerous studies have suggested that the factors that significantly predict visual outcome after OGIs are presenting VA, presence of a relative afferent pupillary defect, mechanism of injury, wound location, wound size and extension, adnexal trauma, lens damage, hyphema, vitreous hemorrhage, and retinal detachment.

- OGIs occur most frequently in males.

- Ruptures are located in the superior half of the eye.

- To our knowledge, there are no studies in the literature about wound characteristics and their effects on final VA in the scleral rupture due to blunt ocular trauma.

What this study adds

- This study has identified associations between wound and ocular characteristics, surgeries and final VA in patients with scleral rupture due to blunt ocular trauma.

- Scleral upture was detected mostly in females, superonasal quadrant and zone 2.

- Poor final VA was significantly associated with poor initial VA, longer wound length, horizontal midline wound, presence of hyphema, vitreous hemorrhage and retinal detachment at presentation, and cataract surgery not performed during follow-up period.

- A moderate positive correlation was found between the initial and final VA and moderate negative correlation was found between wound length and final VA.

\section{Conflict of interest}

The authors declare no conflict of interest.

\section{Acknowledgements}

We thank Naci Murat (Statistician. Assistant Professor. Department of Industrial Engineering, Faculty of Engineering, Ondokuz Mayis University, Samsun, Turkey) for assistance in clinical information analysis.

\section{References}

1 Negrel AD, Thylefors B. The global impact of eye injuries. Ophthalmic Epidemiol 1998; 5: 143-169.

2 Pieramici D, Sternberg P, Aaberg TM, Bridges WZ, Capone A, Cardillo JA et al. A system for classifying mechanical injuries of the eye (globe). The Ocular Trauma Classification Group. Am J Ophthalmol 1997; 123: 820-831. 
3 Rofail M, Lee GA, O'Rourke P. Prognostic indicators for open globe injury. Clin Experiment Ophthalmol 2006; 34(8): 783-786.

4 Al-Mezaine HS, Osman EA, Kangave D, Abu El-Asrar AM. Prognostic factors after repair of open globe injuries. J Trauma 2010; 69(4): 943-947.

5 Yalcin Tök O, Tok L, Eraslan E, Ozkaya D, Ornek F, Bardak Y. Prognostic factors influencing final visual acuity in open globe injuries. J Trauma 2011; 71(6): 1794-1800.

6 Agrawal R, Rao G, Naigaonkar R, Ou X, Desai S. Prognostic factors for vision outcome after surgical repair of open globe injuries. Indian J Ophthalmol 2011; 59(6): 465-470.

7 Rahman I, Maino A, Devadason D, Leatherbarrow B. Open globe injuries: factors predictive of poor outcome. Eye 2006; 20(12): 1336-1341.

8 Agrawal R, Wei HS, Teoh S. Predictive factors for final outcome of severely traumatized eyes with no light perception. BMC Ophthalmol 2012; 12: 16.

9 Yu Wai Man C, Steel D. Visual outcome after open globe injury: a comparison of two prognostic models - the ocular trauma score and the classification and regression tree. Eye 2010; 24(1): 84-89.

10 Feng K, Hu YT, Ma Z. Prognostic indicators for no light perception after open-globe injury: eye injury vitrectomy study. Am J Ophthalmol 2011; 152(4): 654-662.

11 Agrawal R, Ho SW, Teoh S. Pre-operative variables affecting final vision outcome with a critical review of ocular trauma classification for posterior open globe (zone III) injury. Indian J Ophthalmol 2013; 61(10): 541-545.

12 Kim JH, Yang SJ, Kim DS, Kim JG, Yoon YH. Fourteen-year review of open globe injuries in an urban Korean population. J Trauma 2007; 62(3): 746-749.

13 Soni NG, Bauza AM, Son JH, Langer PD, Zarbin MA, Bhagat N. Open globe ocular trauma: functional outcome of eyes with no light perception at initial presentation. Retina 2013; 33(2): 380-386.

14 Emami-Naeini P, Ragam A, Bauza AM, Soni N, Langer P, Zarbin MA et al. Characteristics, outcomes, and prognostic indicators of fall-related open globe injuries. Retina 2013; 33 (10): 2075-2079.

15 Russell SR, Olsen KR, Folk JC. Predictors of scleral rupture and the role of vitrectomy in severe blunt ocular trauma. Am J Ophthalmol 1988; 105(3): 253-257.

16 Cherry PM. Indirect traumatic rupture of the globe. Arch Ophthalmol 1978; 96: 252-256.

17 Knyazer B, Bilenko N, Levy J, Lifshitz T, Belfair N, Klemperer I et al. Open globe eye injury characteristics and prognostic factors in southern Israel: a retrospective epidemiologic review of 10 years experience. Isr Med Assoc J 2013; 15(3): 158-162.

18 Wang YS, Xu JF, Guo CM. Clinical characteristics of occult scleral rupture. Zhonghua Yan Ke Za Zhi 2008; 44(5): 431-435.

19 Kuhn F, Maisiak R, Mann L, Mester V, Morris R, Witherspoon CD. The ocular trauma score (OTS). Ophthalmol Clin North Am 2002; 15: 163-165, vi.

20 Bi H, Cui Y, Li Y, Wang X, Zhang J. Clinical characteristics and surgical problems of ruptured globe injury. Curr Ther Res Clin Exp 2013; 74: 16-21.

21 Salehi-Had H, Andreoli CM, Andreoli MT, Kloek CE, Mukai S. Visual outcomes of vitreoretinal surgery in eyes with severe open-globe injury presentingwith no-light-perception vision. Graefes Arch Clin Exp Ophthalmol 2009; 247(4): 477-483.

22 Boldt HC, Pulido JS, Blodi CF, Folk JC, Weingeist TA. Rural endophthalmitis. Ophthalmology 1989; 96(12): 1722-1726.

23 Thompson WS, Rubsamen PE, Flynn Jr HW, Schiffman J, Cousins SW. Endophthalmitis after penetrating trauma. Risk factors and visual acuity outcomes. Ophthalmology 1995; 102 (11): 1696-1701.

24 Dehghani AR, Rezaei L, Salam H, Mohammadi Z, Mahboubi M. Post traumatic endophthalmitis: incidence and risk factors. Glob J Health Sci 2014; 6(6): 68-72.

25 Lee CH, Lee L, Kao LY, Lin KK, Yang ML. Prognostic indicators of open globe injuries in children. Am J Emerg Med 2009; 27(5): 530-535. 